\title{
Forecasting of Temperature in Bangladesh by using Sliced Functional Time Series (SFTS) Approach
}

\author{
Farhana Akter Bina \\ Lecturer, Department of Statistics, Jahangirnagar University, Savar, Dhaka-1342, \\ Bangladesh. \\ Phone no.: +8801680275349 \\ e-mail: farhanabina@juniv.edu
}

\begin{abstract}
Climate is a paradigm of a complex system and its changes are global in nature. It is an exciting challenge to predict these changes over the period of different time scales. Time series analysis is one of the most important and major tools to analyze the climate time series data. Temperature is one of the most important climatic parameter. In this research, our main aim is to conduct a study across the country to forecast temperature through a relatively new method of forecasting approach named as sliced functional time series (SFTS). The monthly forecasts were obtained along with prediction intervals. These forecasts were compared with the forecasts obtained from autoregressive integrated moving average (ARIMA) and exponential smoothing state-space (ETS) models based on the accuracy measures and the length of prediction intervals to evaluate the performance of SFTS approach.
\end{abstract}

Keywords: Climate,Functional Time Series,Sliced Functional Time Series, Temperature, Forecast, Forecast Accuracy.

\section{Corresponding Author: Farhana Akter Bina}

\section{INTRODUCTION}

Climate change is one of the most important pressing issue for Bangladesh. Climate change of a place refers to the gradual variations in the average weather conditions over a long period of time scales. Bangladesh is highly vulnerable to the impact of these variations as its national economy mainly depends on agriculture and natural resources that are sensitive to climate change and sea level rise.

Bangladesh has a tropical monsoon climate characterized by heavy seasonal rainfall, high temperatures, and high humidity, these are perhaps the most important natural factor. Those factors mostlydetermine the agricultural production and overall economic development of our country. As Bangladesh is an agricultural based country, the amount of seasonal rainfall, extreme level of temperature, its variability and pattern of high or low precipitation affect the country's economic growth mostly.

Since temperature has great influence on climate variability, it is one of the most significant challenge to predict the variability with high accuracy. In this regard time series analysis and forecasting is a major tool to analyze the variability, trends, patterns and to forecast the future pattern. The variability can be modelled accurately using several time series approach. There exists several methods and models to perform time series analysis and forecasting. Several research studies have been developed for the prediction of climate time series using various weather and climate forecasting techniques. 
Besse et al. (2000) examined a class of functional autoregressive (FAR) models which can be used as robust predictors for making forecast of entire smooth functions in future. The methods were illustrated and compared with SARIMA by applying them to forecasting the entire annual cycle climatological ENSO time series one year ahead. Forecasts for the period suggested that the FAR functional predictors show more promising skill compared to traditional scholar SARIMA forecasts which perform poorly (Besse et al.,2000).

Shahid, S. and Khairulmaini, O. S. (2009) studied spatial and temporal variability of rainfall in Bangladesh. They computed long-term annual average rainfall, coefficient of variation of annual rainfall, precipitation concentration and aridity indices at each station and then interpolated them using kriging method within a geographic information system to show the temporal and spatial variability of rainfall. A moderate variation in inter-annual rainfall and high variation in Intra-annual rainfall in Bangladesh have been observed (Shahid, S. and Khairulmaini, O. S.,2009).

Villarini, G. et al. (2010) examined the monthly rainfall and temperature over Rome for the period 1862 to 2004. Application of non-stationary modeling to characterize the nonstationarities in rainfall and temperature data (Villarini, G. et al.,2010).

Shang, H. L. (2011) conducted a study of functional time series approach to forecast short term electricity demand in South Australia. Functional principal component analysis was applied to reduce the dimensionality of curves and penalized least square (PLS) method was used to improve the accuracy of point forecasts. A comparison study was conducted with some naïve benchmark methods and observed that PLS method performs better than any other non-updating methods (Shang, H. L.,2011).

Abbot, J. and Marohasy, J. (2012) analyzed the monthly rainfall in Queensland (Australia) and obtained 3 months ahead forecasts with the application of artificial neural network (ANN). A comparison study was developed between the forecasted and observed rainfall data using time-series plots, root mean squared error (RMSE) and Pearson correlation coefficients (Abbot, J. and Marohasy, J.,2012).

Afsar, S. et al. (2013) proposed a forecasting model that could predict temperature and rainfall and provide timely early warning in Hunza-Nagar. They used the dataset of temperature and rainfall (2007-2011) and developed a quantitative treatment using different statistical methods such as regression and time series/stochastic modeling. The regression analysis proposed that the rainfall increased with increasing temperature. It also found that trends in monthly mean maximum temperature indices increase from years 2007 to 2011 while the amount of rainfall has decreased (Afsar et al., 2013) .

Yasmeen, F. and Sharif, M. (2015) investigated the monthly electricity consumption in Pakistan using functional time series (FTS) technique. 10-year forecasts were obtained and the monthly behavior of forecast values revealed that the electricity consumption will be more for summer season and this demand will be increased in future. Forecast model and the forecast values show that the electricity consumption is increasing with the passage of time (Yasmeen, F. and Sharif, M.,2015).

Alexander et al. (2015) developedd statistical tools for forecasting functional times series which for example can be used to analyze big data sets. They investigtede the impact of time dependence thus quantified on the estimation of functional principal components. After that, they suggested two forecasting techniques for functional time series satisfying measure of time dependence and derive the asymptotic properties of their predictors (Gleim, 2015).

Yasmeen, F. (2016) conducted a research to investigate the breast cancer incidence rates of Karachi (Pakistan) and United States (US) by the application of functional time series (FTS) models and obtained 15 years' predictions of breast cancer incidence rates and showed the future incidence rates will be expected to rise only for those aged 50 years and above, but the rates remained stable for the women younger than 50 years (Yasmeen, F.,2016). 
Yamoah et al. (2016) studied the rainfall pattern for the period of 1975 to 2009 in the BrongAhafo (BA) region of Ghana. Application of SARIMA modelling and forecasting methods to

examine the rainfall pattern and fit a suitable model for rainfall prediction. An appropriate model SARIMA $(0,0,0) \times(1,1,1)_{12}$ was identified for predicting monthly average rainfall for the region (Yamoah et al.,2016).

Yasmeen, F. and Hameed, S. (2018) conducted a study with a new method of forecasting sliced functional time series (SFTS) approach. They analyzed rainfall trend in Pakistan over the past six decades and obtain the next ten years' forecasts along with $80 \%$ prediction intervals. These forecasts were also compared with the forecasts obtained from ARIMA and exponential smoothing state space (ETS) models (Yasmeen, F. and Hameed, S.,2018).

Yasmeen, F. and Hameed, S. (2019) examined the combined land-surface air and sea-surface water temperature from Goddard Institute for Space Studies (GISS), NASA using sliced functional time series (SFTS) model. They analyze the global surface temperature change, compare alternative analyses, and address the questions about the reality of global warming and also detected the outliers during the last century not only in global temperature series but also in northern and southern hemisphere series. The forecasts for the next twenty years were obtained using SFTS models and observed that SFTS models performed better than the other models with narrow prediction intervals (Yasmeen, F. and Hameed, S.,2019).

From the above discussion it is observed that, to examine the future projections of climate time series there exists several methods and models. In this study the variation and characteristics of monthly average temperature over the specified region of Bangladesh will be examined and the main objective is to apply the sliced functional time series (SFTS) approachto obtain the forecasts from SFTS models. To make comparisons, forecasts will also be obtained from some other models using exponential smoothing state-space (ETS) models and autoregressive integrated moving average ARIMA models. Finally, the comparisons will be made among the different models based on forecasts accuracy to evaluate the performance of SFTS models.

\section{DATA DESCRIPTION AND STUDY AREA}

Bangladesh has mainly three seasons: The Monsoon or wet season from late May to early October, the cold season from mid-October to the end of February, and the summer season from mid-march to mid-May. There are widespread differences in the intensity of the seasons at different places of the country. On the basis of entire climatic condition Bangladesh is divided into seven distinct climatic zones-South-Eastern Zone, North-Eastern Zone, Northern Part of the Northern Region, North-Western Zone, Western Zone, South-Western Zone, South-Central Zone(Climatic Zone - Banglapedia).

The Figure 1 depicts the different climate zones and selected districts under each zone. From the figure, Dhaka station falls into the south-central zone. In this zone rainfall is abundant, being above $1,900 \mathrm{~mm}$. The range of temperature is much less than to the west, but somewhat more than in South-eastern zone. This is a transitory zone between the South-eastern, Northwestern and South-western zones and most of the severe hail storms, nor 'westers and tornadoes are recorded in this area (Climatic Zone - Banglapedia).

To carry out the research analysis, data on monthly average dry-bulb Temperature $\left(\right.$ in ${ }^{\circ} \mathrm{C}$ ) of Dhaka station (study area) for the last 50 years in Bangladesh was obtained from Bangladesh Meteorological Department (BMD). The data includes monthly average dry-bulb temperature (in ${ }^{\circ} \mathrm{C}$ ) for the period from January 1968 to December 2019 for the selected study area. 


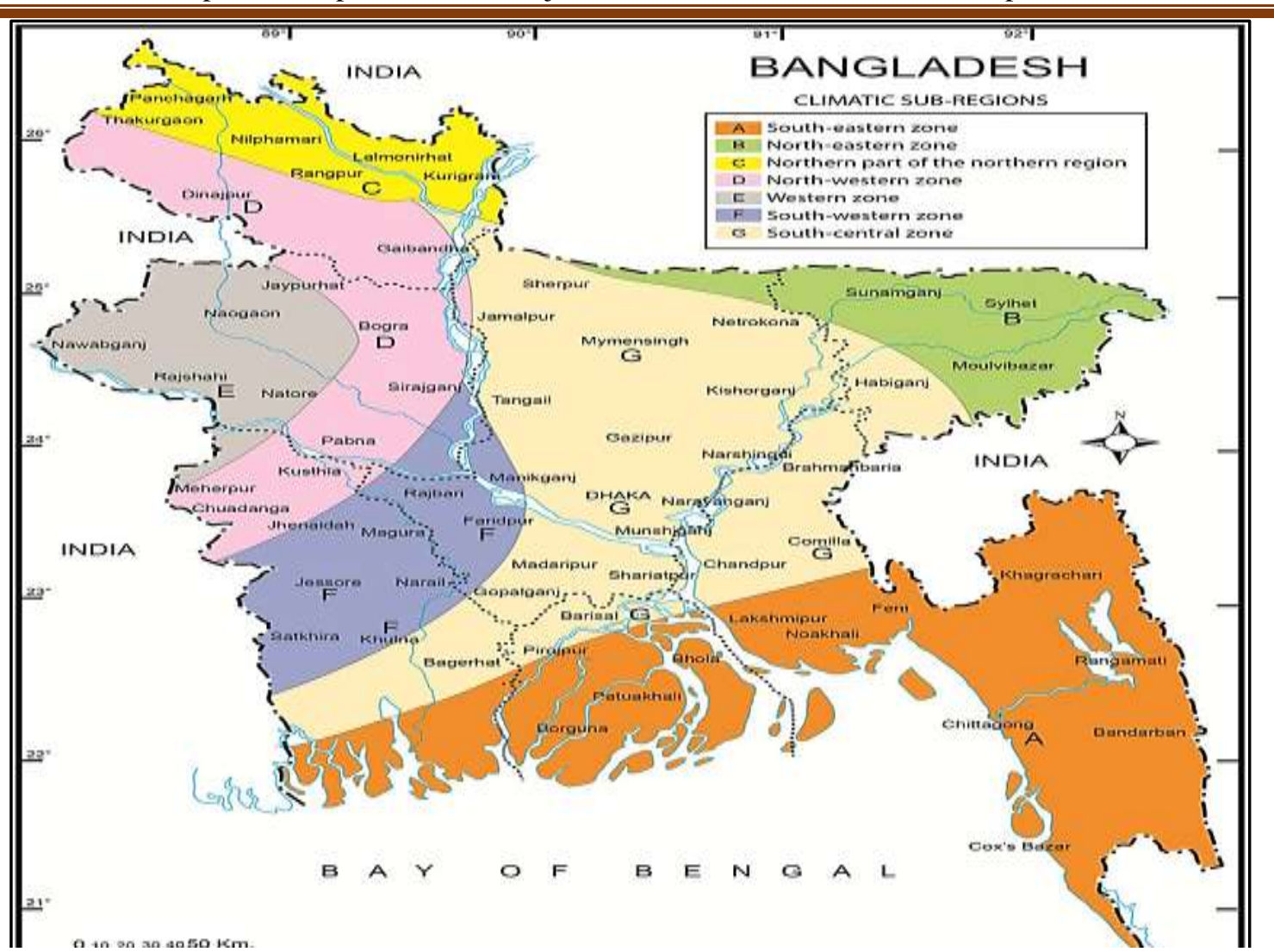

Figure 1: Climate Zone Map of Bangladesh (Sarker et al., 2019).

\section{MATERIALS AND METHODS}

\subsection{Functional Time Series (FTS) and Sliced Functional Time Series (SFTS)}

Hyndman and Ullah (2007) first introduced the functional time series (FTS) models which can be defined as a time sequence of functional observations i.e. the realization of a functional stochastic process in time (González J. P., 2017). Hyndman and Ullah (2007), Shang H. L. (2011) and Yasmeen et al. (2016) used functional time series (FTS) for forecasting purposes. Initially the FTS approach was applied to model the log mortality and fertility rates.In recent times, functional time series models have been applied for demographic forecasting and for mortality forecasting. Also these models are used in forecasting the mortality rates of chronic diseases such as breast cancer (Yasmeen and Sharif, 2015). Using this method, the functional curves are observed (with error) at time $t=$ $1,2, \cdots, m$, and we wish to forecast the functions for times $t=m+1, \cdots, m+h$. Let $\left[g_{t}\left(x_{j}\right)\right]$ denote the observed data, where $j=1, \cdots, p$. We assume that there are underlying $L_{1}$ continuous and smooth functions $\left[f_{t}(x)\right]$ such that

$$
g_{t}\left(x_{j}\right)=f_{t}\left(x_{j}\right)+\delta_{t}\left(x_{j}\right) e_{t, j},
$$

where $e_{i j}$ are independent and identically distributed random variables with zero mean and unit variance, and $\delta_{t}\left(x_{j}\right)$ allows for heteroscedasticity (Yasmeen and Hameed, 2018).

The Hyndman and Ullah (2007) technique uses non parametric smoothing on each curve $g_{t}(x)$ separately to obtain estimates of the smooth functions $\left[f_{t}(x)\right]$. Panelized regression splines (Wood, 1994) are used for smoothing, and then a functional principal component approach (Ramsay and Silverman, 2005) is used to decompose the time series of functional data into a number of principal components and their scores. The functional time series (FTS) model can be written as follows:

$$
f_{t}(x)=\mu(x)+\sum_{k=1}^{K} \alpha_{t, k} \Psi_{k}(\mathrm{x})+e_{t}(x),
$$


where $\Psi_{k}(\mathrm{x})$ is the $k^{t h}$ principal component, the set of coefficients $\left[\alpha_{1, k}, \cdots, \alpha_{m, k}\right]$ are the corresponding scores, $e_{t}(x)$ denotes the independent and identically distributed random functions with zero mean, and $K$ is the number of principal components to be used (Yasmeen and Hameed, 2018).

The first step is to obtain the functional curves and then the data are transformed into sliced functional time series (SFTS) to detect the outliers from a functional time series. The entire data are sliced for each year as a function of 12 months. These curves are plotted in rainbow order with red for the earlier years and violet for the most recent years. The functional curves are then projected into a finite dimensional subspace $\left(R^{2}\right)$ where each of the data points are ordered by data depth and data density based on different graphical methods (e.g. Halfspace Bagplot, HDR Boxplot). The outliers can be identified from those curves having either lowest depth and/or lowest density (Hyndman and Shang, 2010).

\subsection{Outlier Detection}

Identification of outliers in a dataset is very important because the presence of outliers may have a serious effect on modeling and forecasting series.Statistical analysis which does not involve identifying outliers can often lead to inaccurate conclusions. Despite the obvious importance of this problem, we are aware of only two previous approaches to functional outlier detection. Hyndman and Ullah (2007) used a method based on Robust Principal Component Analysis, while Febrero et al. (2007, 2008) considered Functional Outlier Detection using successive likelihood ratio tests and smoothed bootstrapping (Hyndman and Shang, 2010).

The functional data analytic toolbox proposed by Hyndman and Shang (2010) which can be used for visualizing large numbers of functional data in the form of smooth curves or surfaces. These new graphical displays are outlier detection methods for functional data. They are-
i. Rainbow Plot
ii. Functional Bagplot
iii. Functional Highest Density Region (HDR) Plot.

Those graphical methods have the benefits to identify the outliers which may not be obvious from a plot of the original data. Outlying curves may either lie outside the range of the vast majority of the data (we call these "magnitude outliers"), or they may be within the range of the rest of the data but have a very different shape from other curves (we call these "shape outliers"), or they may exhibit a combination of these features.

\subsubsection{Rainbow Plot}

The rainbow plot is a simple plot of all the data, with the only added feature being a color palette based on an ordering of the data with red for the earlier years and violet for the most recent years.It may also be difficult to see outliers if they are obscured by other curves (for example, curves having a different shape from the rest of the data).

For data that are not naturally ordered by time, or some other index, the rainbow plot can still be used by constructing an ordering index such as the data depth or data density indexes defined above. Then the colors are chosen in rainbow order according to the ordering of $\left\{\sigma_{i}\right\}$. If there is no time trend in the dataset, then a rainbow plot with time ordering is not particularly informative (Hyndman and Shang, 2010).

\subsubsection{Functional Bagplot}

The functional bagplot is based on the bivariate bagplot of Rousseeuw et al. (1999), applied to the first two principal component scores which uses Tukey's halfspace location depth 
(Yasmeen, 2019). The depth region $D_{k}$ is the set of all $\theta$, with $d(\theta, z) \geq k$. Since the depth regions form a series of convex hulls, we have $D_{K_{1}} \subset D_{K_{2}}$ for $K_{2}>K_{1}$. The Tukey bivariate depth median is defined as the value of $\theta$ which minimizes $d(\theta, Z)$ if there is such a unique $\theta$; otherwise it is defined as the center of gravity of the deepest region.

Like a univariate boxplot, the bivariate bagplot has a central point (the Tukey median), an inner region (the 'bag') and an outer region, beyond which outliers are shown as individual points. The bag is defined as the smallest depth region containing at least $50 \%$ of the total number of observations. The outer region (or 'fence') of the bagplot is the convex hull of the points contained within the region obtained by inflating the bag (relative to the Tukey median) by a factor $\rho$.

The functional bagplot is a mapping of the bagplot of the first two robust principal component scores to the functional curves. The functional bagplot displays the median curve (the curve with the greatest depth), and the inner and outer regions. The inner region is defined as the region bounded by all curves corresponding to points in the bivariate bag. Thus, $50 \%$ of curves are in the inner region. The outer region is similarly defined as the region bounded by all curves corresponding to points within the bivariate fence region. The functional bagplot may be a good outlier detection method when outliers are far away fromthe median. However, when outliers are near the median, this depth-measure outlier detection tool can misidentify outliers. In this situation, the functional HDR boxplot is more appropriate.

\subsubsection{Functional HDR Boxplot}

A boxplot may also indicate which observations, if any, can be considered as outliers. The functional HDR boxplot is based on the bivariate HDR boxplot (Hyndman, 1996), which is applied to the first two principal component scores. Constructing a Functional HDR boxplot consists of some particular steps:

i. Compute a multivariate kernel density estimation,

ii. Compute contour lines for quantiles $90 \%, 50 \%$ and alpha $\%$,

iii. Plot the bivariate plot, and

iv. Compute median curve along with quantiles and outliers' curves.

The bivariate HDR boxplot is constructed using a bivariate kernel density estimate $f(z)$, which is defined as

$$
f(z)=1 / n \sum_{i=1}^{n} K_{h_{i}}\left(z-Z_{i}\right)
$$

where, $Z_{i}$ represents a set of bivariate points, $K_{h_{i}}(\cdot)=K\left(\cdot / h_{i}\right) / h_{i}$, $\mathrm{K}$ is the kernel function, and $h_{i}$ is the bandwidth for the $i t h$ dimension. The bandwidths were selected using smoothed cross validation (Dunong, 2005). Using kernel density estimates, HDR can be defined as

$$
R_{\alpha}=\left\{z:(z) \geq f_{\alpha}\right\}
$$

For $f_{\alpha}$ is such that $\int_{R_{\alpha}} f(z) d z=1-\alpha$; that is, it is the region with probability coverage $1-\alpha$, where all points within the region have a higher density estimate than any of the points outside the region - hence the name "highest density region". For a bivariate density, the HDRs can be considered as contours, with an expanding coverage as $\alpha$ decreases.

The bivariate HDR boxplot displays the mode, defined as $\arg \sup f(z)$, along with the $50 \%$ inner and (usually) 99\% highest density regions. All points excluded from the outer HDR are outliers. The functional HDR boxplot is a mapping of the bivariate HDR boxplot of the first two robust principal component scores to the functional curves. The functional HDR boxplot displays the modal curve (the curve with the highest density), and the inner and outer regions. The inner region is defined as the region bounded by all curves corresponding to points inside the $50 \%$ bivariate HDR. Thus, $50 \%$ of curves are in the inner region. The outer region is 
similarly defined as the region bounded by all curves corresponding to the points within the outer bivariate HDR (Hyndman and Shang, 2010).

\subsection{The h-step-ahead Forecasts}

The h-step-ahead forecasts of $Y_{n+h}(x)$ can be obtained as:

$$
\widehat{\mathrm{Y}}_{\mathrm{n}+\mathrm{h} / \mathrm{n}}=\hat{f}(x)+\sum_{K=1}^{K} \hat{\alpha}_{n+h /(n, j)} \widehat{\Psi}_{K}(x)+e_{t},
$$

where, $\hat{\alpha}_{n+h /(n, j)}$ shows the h-step-ahead forecasts of $\alpha_{n+h /(n, j)}$ using univariate time series and $\widehat{\Psi}_{K}(x)$ are the estimated basis function obtained by functional principal component analysis (Hyndman and Ullah, 2007).

\subsection{Forecast Errors}

A forecast "error" is the difference between an observed value and its forecast. Here "error" does not mean a mistake, it means the unpredictable part of an observation. It can be written as:

$$
e_{t}=y_{t}-\hat{y}_{t}
$$

where $e_{t}$ is the forecast error at period $t, y_{t}$ is the actual value at period $t$, and $\hat{y}_{t}$ is the forecast for period $t$. There are different measures of forecasting errors which are used to check the forecasting performance (Hyndman and Koehler, 2006).

i. Mean Error (ME): $M E=\operatorname{mean}\left(\left|e_{t}\right|\right)$.

ii. Mean Squared Error (MSE): $M S E=\operatorname{mean}\left(e_{t}^{2}\right)$.

iii. Root Mean Squared Error (RMSE):RMSE $=\sqrt{\operatorname{mean}\left(e_{t}^{2}\right)}$.

iv. Mean Percentage Error (MPE): $M P E=\operatorname{mean}\left(p_{t}\right)$, where $p_{t}=100 e_{t} / y_{t}$.

v. Mean Absolute Percentage Error (MAPE):MAPE $=$ mean $\left(\left|p_{t}\right|\right.$.

\section{RESULTS AND DISCUSSIONS}

\subsection{Time Series Analysis}

Figure 2 represents the monthly plot of average temperature $\left({ }^{\circ} \mathrm{C}\right)$ of Dhaka (1968-2019) which shows a clear seasonal pattern. The average monthly maximum temperature was $30.7^{\circ} \mathrm{C}$ in June 1998 and in April 2014 and the average monthly minimum temperature was $16.2^{\circ} \mathrm{C}$ in December 2003. In 2019 the average monthly maximum temperature was $29.9^{\circ} \mathrm{C}$ in June and August, the minimum was $19.3^{\circ} \mathrm{C}$ in December. According to the historical data the warmest month is April and coolest month is January.

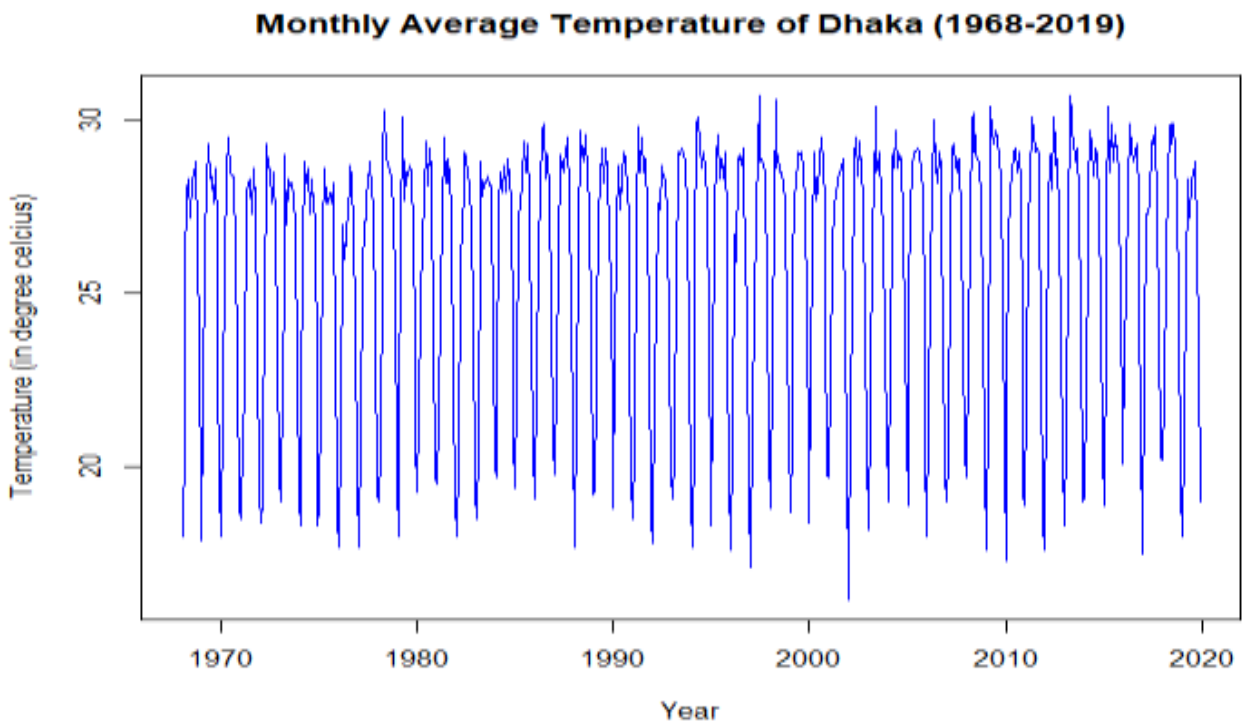

Figure 2: Time series plot of monthly average temperature data of Dhaka (1968-2019). 
4.2. Sliced Functional Time Series (SFTS) Analysis

Figure 3 represents sliced functional time series of temperature data. For this, the data was transformed into sliced functional time series. To obtain the functional curves, the entire data was sliced for each year as a function of 12 months. These curves are plotted in rainbow order with red for the earlier years and violet for the most recent year.

Figure 4 represents the season plots by extracting the sliced functional time series by order of time. For this plot "rainbow" (Shang, H. L. and Hyndman, R. J., 2016), "ftsa" (Hyndman, R. J. and Shang, H. L., 2019) and "fts" (Armstrong, W., 2018) packages in R statistical software (Hyndman and Shang, 2009) were used.

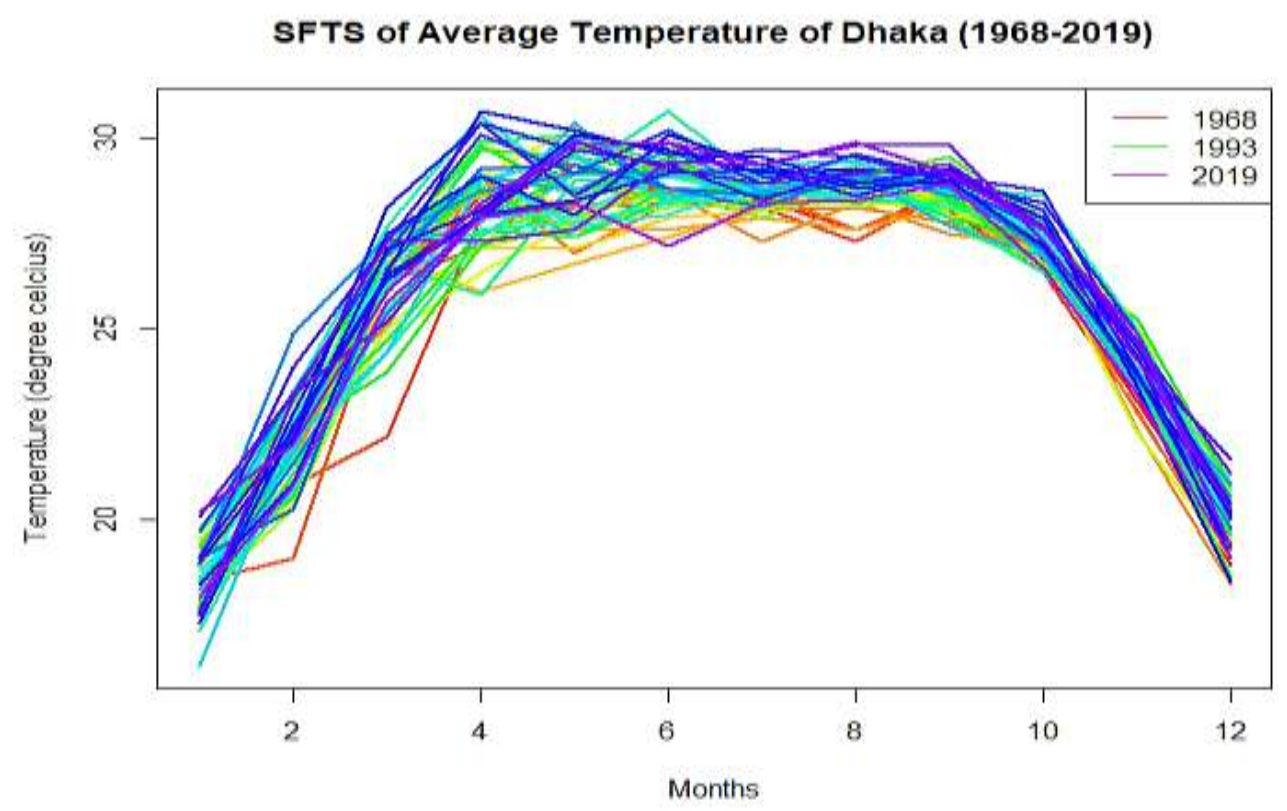

Figure 3: Sliced functional time series (SFTS) of monthly temperature data of Dhaka (1968-2019).
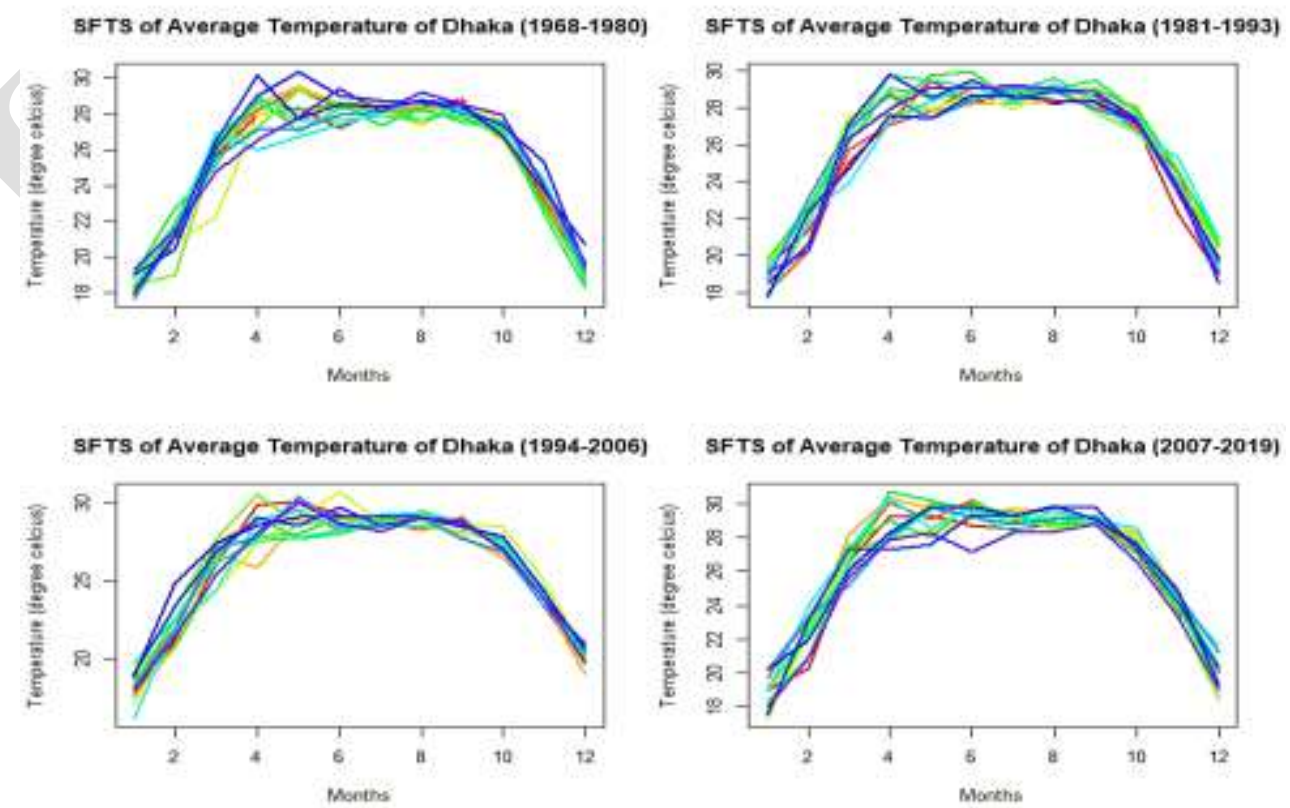

Figure 4: Season plots of temperature data during (1968-1980), (1981-1993), (19942006), and (2007-2019). 


\subsection{Outlier Detection}

As discussed in the methodology section, for outlier detection the functional curves were projected into a finite dimensional subspace $\left(R^{2}\right)$. In this subspace, each of the functional data points were ordered by data depth and data density. Lowest depth or lowest density will be considered to be the outliers.

\subsubsection{Functional Bagplot}

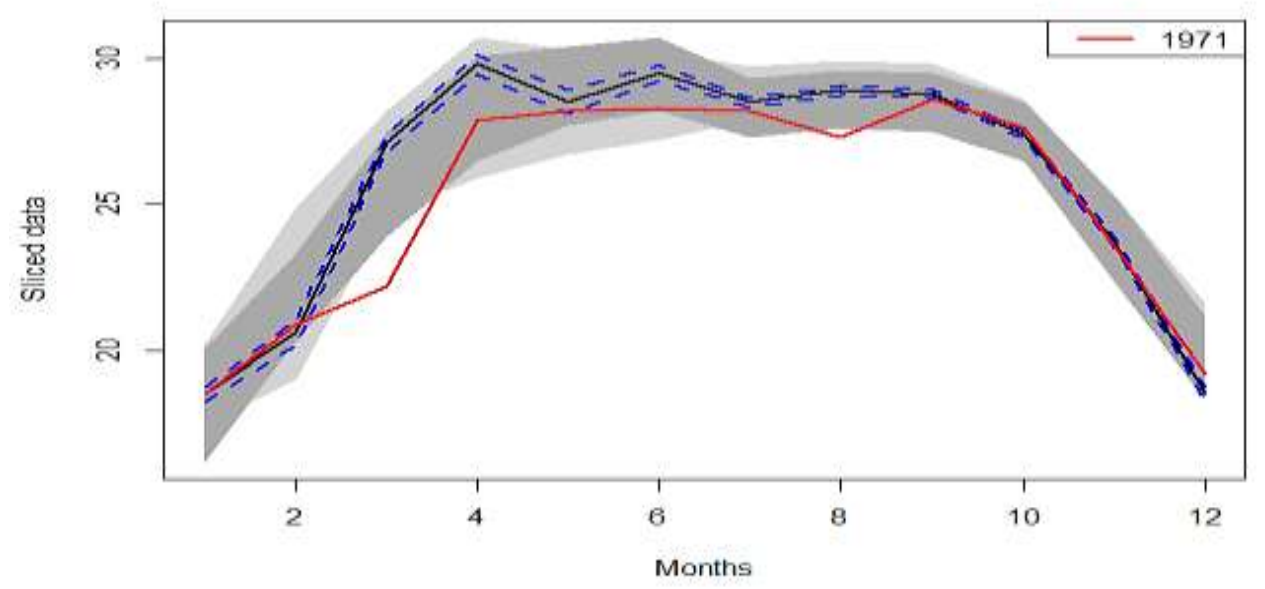

Figure 5: Functional bagplot of monthly temperature data of Dhaka (1968-2019).

The functional bagplot of sliced functional time series of temperature data are plotted in Figure 5 where the median curve is denoted by black color along with blue dotted confidence interval. The outliers are represented by red color. Inner and outer regions are plotted with dark grey and light grey colors respectively.

\subsubsection{Functional HDR Plot}

Figure 6 represents the functional HDR boxplots for the sliced functional time series, where the modal curve is denoted by black color and the outliers are represented by red, green and blue colors. Inner and outer regions are plotted with dark grey and light grey colors respectively.

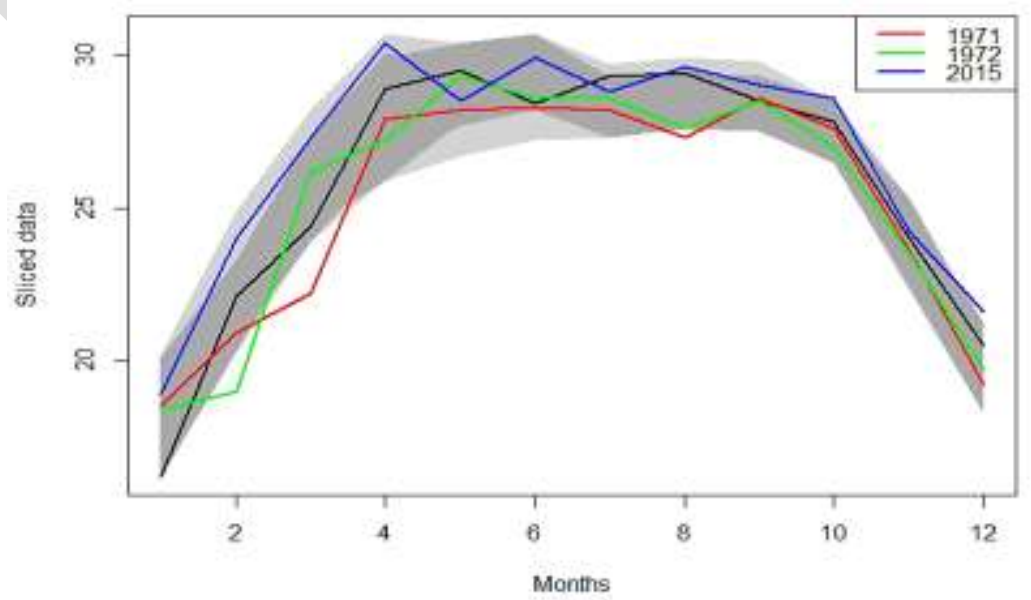

Figure 6: Functional HDR plot of monthly temperature data of Dhaka (1968-2019). 


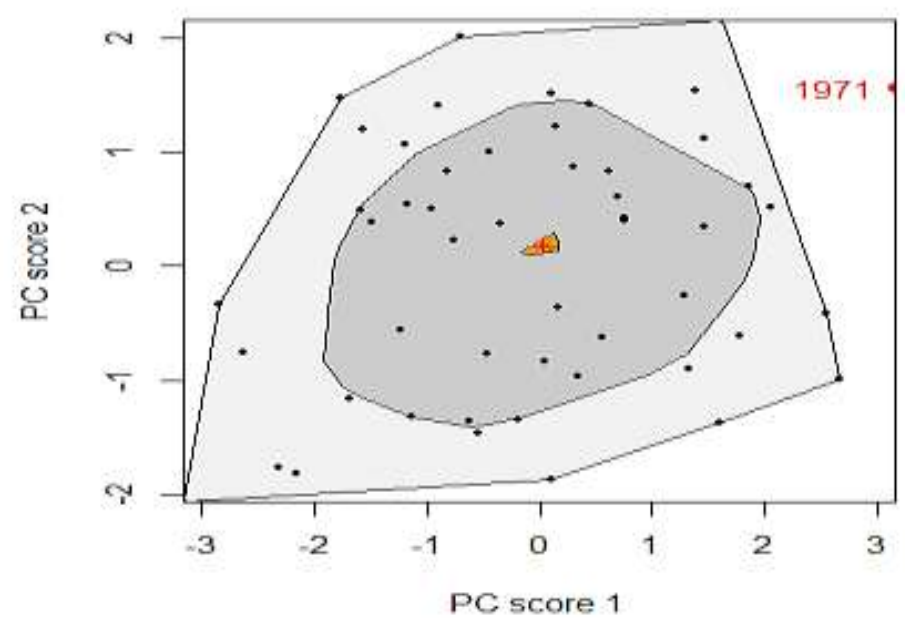

Figure 7: Functional bivariate plot of monthly temperature data of Dhaka (1968-2019) with first two principal components.

Lastly, the functional bivariate plots with first two principal components are constructed in Figure 7. The red asterisk is the sample median and the inner and outer regions are plotted with dark grey and light grey colors respectively. 1971 is the confirmed outlier from all three methods of the respective time series.

Table 1: Outliers in temperature series (1968-2019) using different outlier detection methods.

Method

Outliers of Temperature Series

$\begin{array}{lc}\text { Functional Bagplot } & 1971 \\ \text { Functional HDR plot } & 1971,1972,2015 \\ \text { Functional Bivariate plot } & 1971\end{array}$

\subsection{Forecasting with FTS Model}

Next, a functional time series model (FTSM) is applied to obtain the next twenty years (20202039) forecasts of the sliced functional time series. The mean function and different components (basis functions, corresponding time series coefficients) of FTS model are plotted with twenty years' forecasts and their $80 \%$ prediction interval (in yellow and green color respectively), using an exponential smoothing state-space model (Hyndman et al., 2008) in Figure 8.

From the Figure 8, the first function shows that the month April is more responsible for the variation and the forecast shows an increasing trend in future. The other two functions represent the months February and April and their forecasts (in red color) shows steady behavior with $80 \%$ prediction intervals (in yellow color). Other functions and their forecasts are not plotted as they are more complicated and relatively less important. 

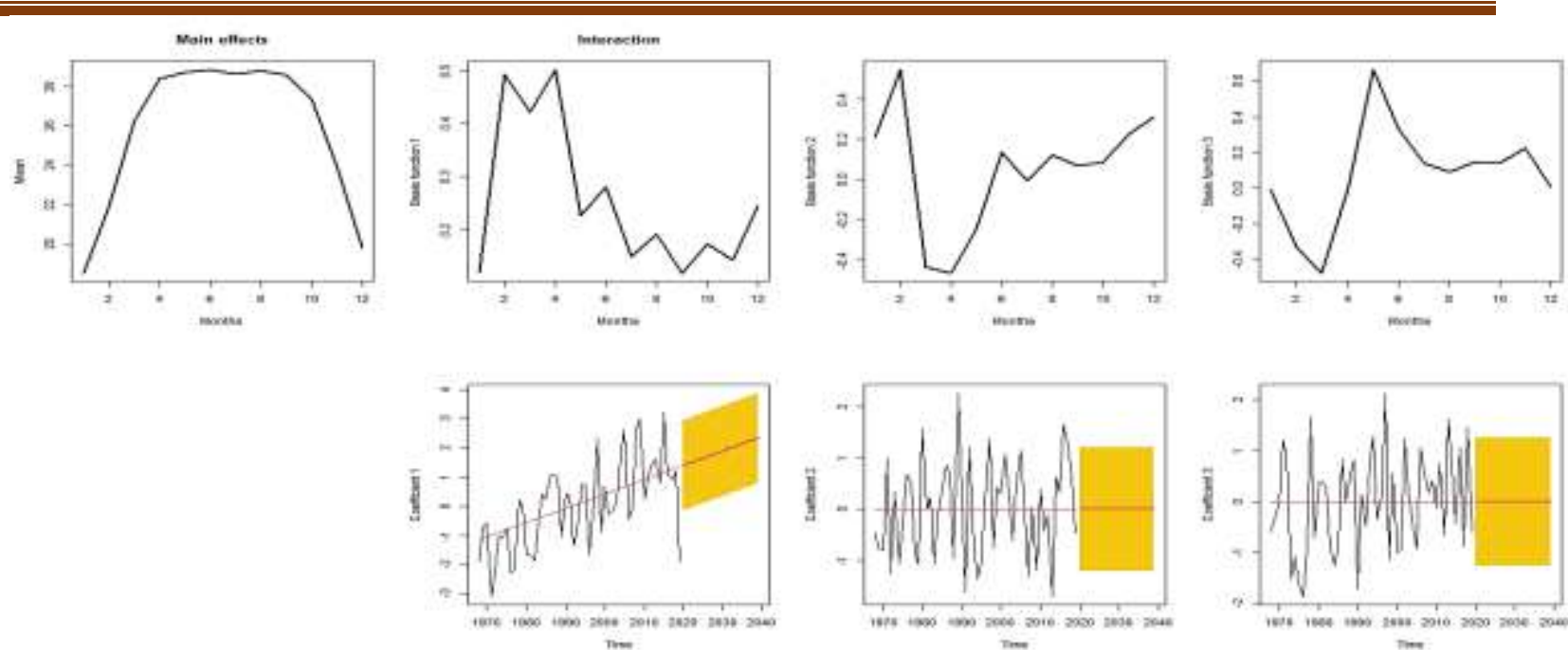

Figure 8: Different components of FTS models applied to the monthly temperature data of Dhaka (1968-2019), along with 20-year forecasts and $80 \%$ prediction intervals of the time series coefficients.

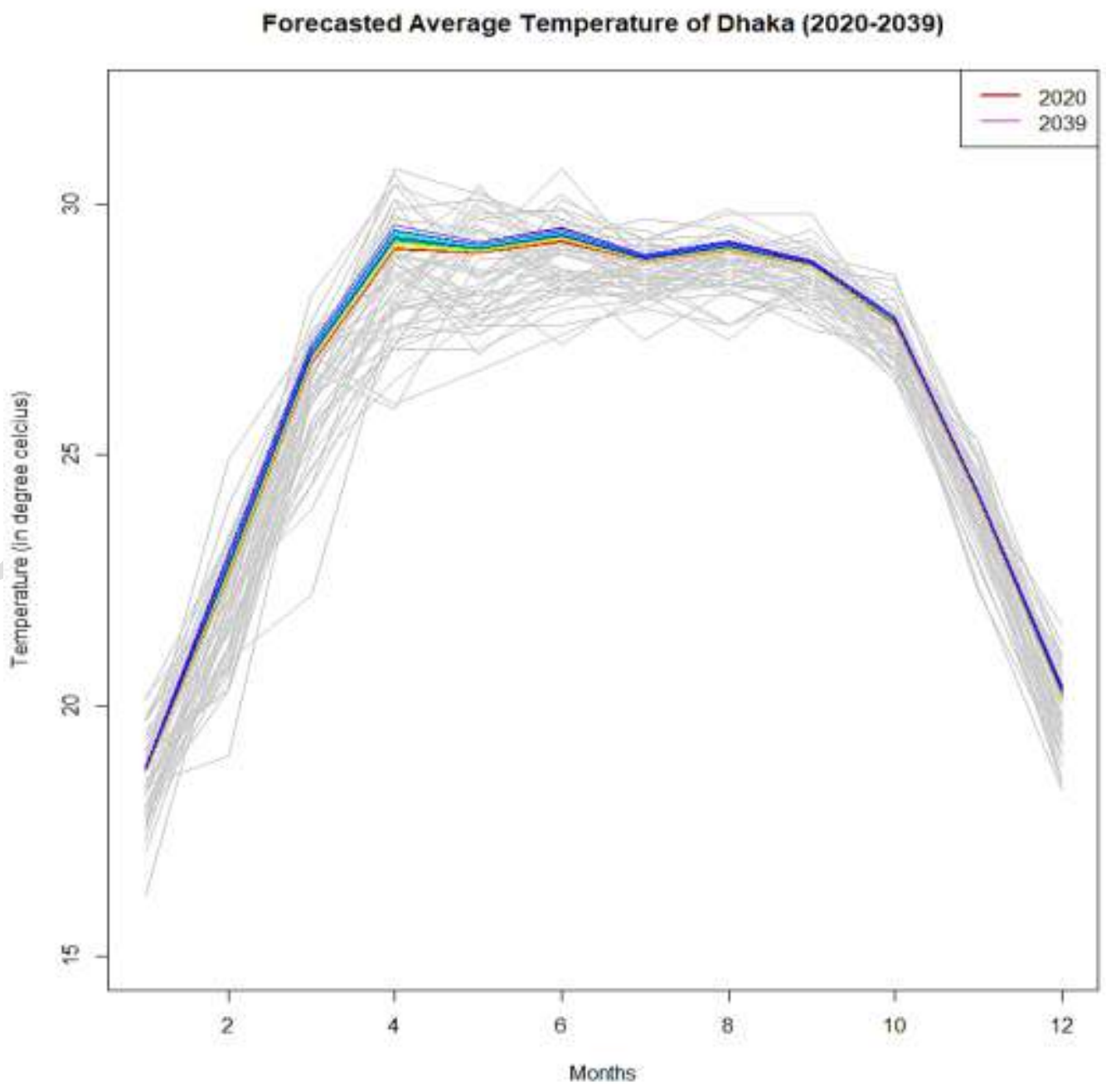

Figure 9: SFTS forecasts of monthly average temperature of Dhaka (2020-2039) based on historical data (1968-2019). 
Figure 9 shows the forecasts of average temperature of Dhaka from 2020 to 2039 highlighted in rainbow color, with red for the most recent years and violet for the future years and the historical data used for estimation are grayed out.

\subsection{Forecasting with Other Models}

Figure 10 represents the forecasts of next 20 years (2020-2039) obtained from Exponential Smoothing State-Space (ETS) model $\operatorname{ETS}(A, N, A)$ along with $80 \%$ prediction intervals. The red color represents the forecasted series and the green color represents the $80 \%$ prediction intervals.

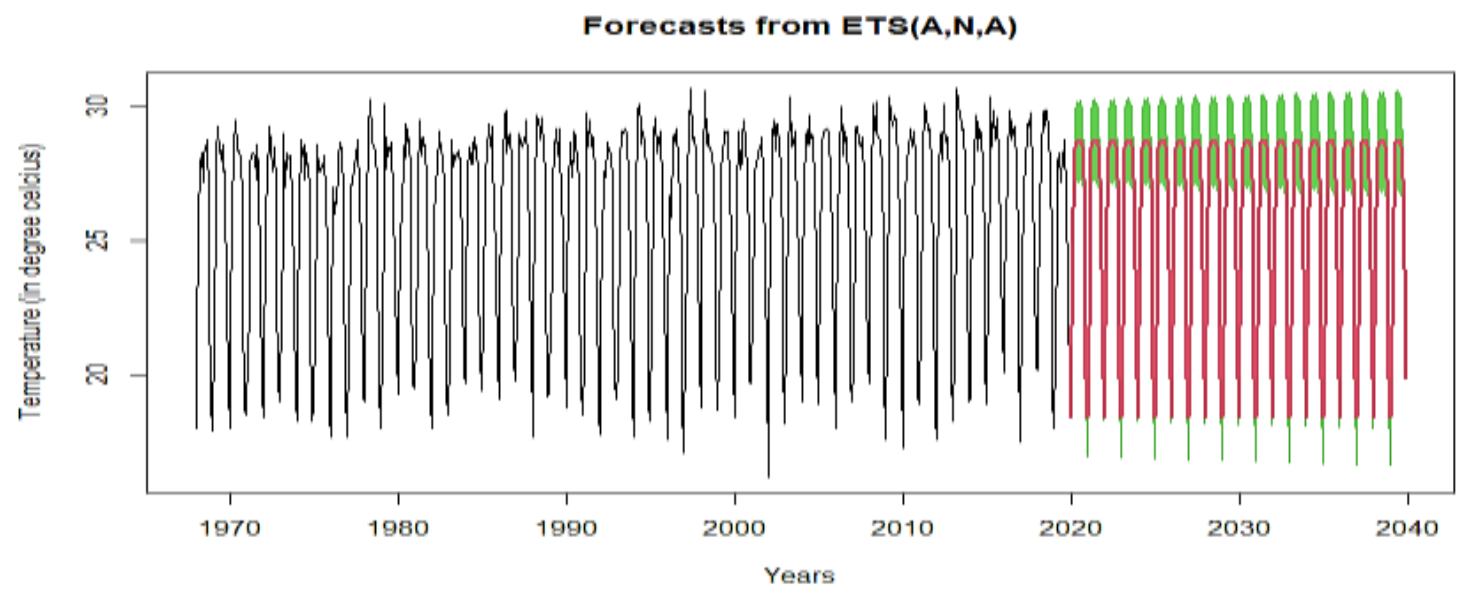

Figure 10: Forecasts of monthly average temperature of Dhaka (2020-2039) using ETS model.

Figure 11 represents the forecasts of next 20 years (2020-2039) obtained from ARIMA model ARIMA $(2,0,1)(2,1,0)[12]$ with drift with $80 \%$ prediction intervals. The red color represents the forecasted series and the green color represents the $80 \%$ prediction intervals.

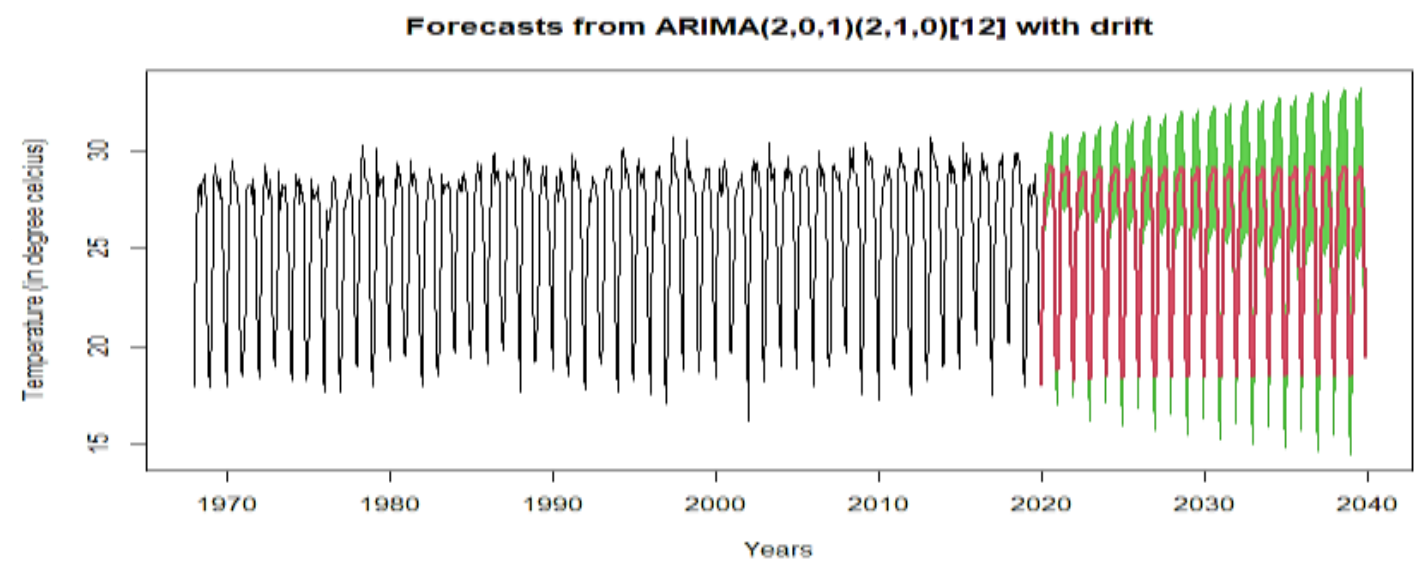

Figure 11: Forecasts of monthly average temperature of Dhaka (2020-2039) using ARIMA model.

Table 2: Forecasting performance of different models of temperature data.

\begin{tabular}{lccccc}
\hline Models & ME & MSE & RMSE & MPE & MAPE \\
\hline ETS & 0.01267 & 0.56632 & 0.75254 & -0.04143 & 2.30366 \\
ARIMA/SARIMA & 0.00259 & 0.71064 & 0.84299 & -0.08042 & 2.52963 \\
SFTS & -0.00030 & 0.35153 & 0.59289 & 0.00065 & 0.01856 \\
\hline
\end{tabular}


Table 3: Forecasts of monthly average temperature of Dhaka obtained from ETS, ARIMA and SFTS models.

\begin{tabular}{|c|c|c|c|c|}
\hline \multirow[b]{2}{*}{ Year } & \multirow[b]{2}{*}{ Time } & \multicolumn{3}{|c|}{$\begin{array}{l}\text { Forecasted Average } \\
\text { Temperature }\left({ }^{\circ} \mathrm{C}\right)\end{array}$} \\
\hline & & ETS & ARIMA & SFTS \\
\hline \multirow{12}{*}{2020} & January & 18.44 & 18.04 & 18.68 \\
\hline & February & 21.83 & 21.82 & 22.56 \\
\hline & March & 26.23 & 26.18 & 26.82 \\
\hline & April & 28.45 & 27.63 & 29.10 \\
\hline & May & 28.70 & 28.35 & 29.03 \\
\hline & June & 28.81 & 28.70 & 29.26 \\
\hline & July & 28.64 & 28.87 & 28.85 \\
\hline & August & 28.78 & 29.28 & 29.07 \\
\hline & September & 28.54 & 29.19 & 28.75 \\
\hline & October & 27.34 & 27.1 & 27.59 \\
\hline & November & 23.99 & 24.06 & 24.14 \\
\hline & December & 19.85 & 19.53 & 20.15 \\
\hline \multirow{12}{*}{2021} & January & 18.44 & 18.80 & 18.69 \\
\hline & February & 21.83 & 21.59 & 22.59 \\
\hline & March & 26.23 & 25.95 & 26.84 \\
\hline & April & 28.45 & 27.95 & 29.13 \\
\hline & May & 28.70 & 28.86 & 29.04 \\
\hline & June & 28.81 & 28.69 & 29.28 \\
\hline & July & 28.64 & 28.86 & 28.86 \\
\hline & August & 28.78 & 29.25 & 29.08 \\
\hline & September & 28.54 & 29.04 & 28.76 \\
\hline & October & 27.34 & 27.14 & 27.61 \\
\hline & November & 23.99 & 24.15 & 24.15 \\
\hline & December & 19.85 & 19.29 & 20.16 \\
\hline \multirow{12}{*}{2022} & January & 18.44 & 18.29 & 18.69 \\
\hline & February & 21.83 & 21.41 & 22.6 \\
\hline & March & 26.23 & 25.94 & 26.86 \\
\hline & April & 28.45 & 27.84 & 29.15 \\
\hline & May & 28.70 & 28.51 & 29.05 \\
\hline & June & 28.81 & 28.15 & 29.29 \\
\hline & July & 28.64 & 28.67 & 28.87 \\
\hline & August & 28.78 & 28.96 & 29.09 \\
\hline & September & 28.54 & 29.01 & 28.77 \\
\hline & October & 27.34 & 26.94 & 27.61 \\
\hline & November & 23.99 & 23.82 & 24.16 \\
\hline & December & 19.85 & 19.27 & 20.17 \\
\hline
\end{tabular}

Forecasts obtained from FTS model in the previous section are compared with the forecasts obtained from ETS and ARIMA models by measuring different forecasting performance (ME, MSE, RMSE, MPE, MAPE etc.) which are represented in Table 2. It is found that SFTS models performs better than ETS and ARIMA models as the SFTS model has smaller ME, MSE, RMSE and MAPE than the others. So SFTS model is a good choice to use for forecasting purpose comparatively than other models. The forecasted values from different models are shown in Table 3. 


\section{CONCLUSION}

Functional time series models have been applied in recent times for demographic and mortality forecasting. These models have been used in forecasting a series of functional data observed over a period of time. Sliced functional time series (SFTS) approach is a new forecasting technique to forecast functional data by slicing them. The SFTS approach was applied to forecast the selectedclimate time series and to examine the performance of SFTS models which was the main objective of this research study.

The analysis was carried out using the univariate time series data of monthly average drybulb temperature $\left({ }^{\circ} \mathrm{C}\right)$ (1968-2019) of Dhaka which was obtained from Bangladesh Meteorological Department. The data was analyzed through sliced functional time series (SFTS) analysis. The entire data was sliced and plot of functional curves were obtained, where each year showing a function of 12 months in a rainbow order. Using functional bagplot, functional HDR boxplot and functional bivariate plots, outliers were detected. Finally, using functional time series model, a 20-years ahead forecasts (2020-2039) of the sliced functional time series were obtained along with $80 \%$ prediction intervals. These forecasts were compared with the forecasts obtained from exponential smoothing state-space (ETS) models and autoregressive integrated moving average (ARIMA) models. Forecasting performance of different models were measured and compared. It was found that SFTS models performed better and forecasts were more accurate and reliable than the ETS and ARIMA models.

In short, it is always in demand that a better forecast depends on better model. Finding such models under realistic situations is more challenging. In this research study it is obtained that SFTS models performed better over the other models for the selected series. So we can conclude that sliced functional time series (SFTS) modelscan be a good choice for forecasting purposewhich fulfills the aim of this research.

\section{DECLARATION OF COMPLETING INTEREST}

The author declares no conflicts of interest regarding the publication of this paper.

\section{ACKNOWLEDGEMENT}

The author is grateful to the University Grants Commission of Bangladesh for funding this research work and also thankful to Bangladesh Meteorological Department for providing data.

\section{REFERENCES}

1. Ahmed, B., Kelman, I., Kamruzzan, M. and Mohiuddin, H. (November 2018). Indigenous People's Responses to Drought in Northwest Bangladesh.Journal of Environmental Development, ISSN 2211-4645.

2. Afsar, S., Abbas, N., and Jan, B. (2013). Comparative Study of Temperature and Rainfall Fluctuation in Hunza-Nagar Comparative Study of Temperature and Rainfall Fluctuation in Hunza-Nagar District. Journal of Basic and Applied Sciences, 9, 151156.https://doi.org/10.6000/1927-5129.2013.09.21.

3. Abbot, J. and Marohasy, J. (2012). Application of Artificial Neural Networks to Rainfall in Queensland, Australia. 29(4), 717-730.

4. Armstrong, W. (2018). R Interface to 'tslib' (a Time Series Library in C++), Package 'fts'. Version 0.9.9.2.

5. Besse, P. C., Cardot, H. and Stephenson, D. B. (2000). Autoregressive Forecasting of Some Functional Climatic Variations.Scandinavian Journal of Statistics.27(1993), 673687.

6. Braun, Maxwell, D. (20 October 2010). Bangladesh, India Most Threatened by Climate 
Change, Risk Study Finds. National Geographic.

7. Climatic Zone-Banglapedia. National Encyclopedia of

Bangladesh.http://en.banglapedia.org/index.php?title=Climate.

8. Dunong, T. (2005). Bandwidth Selectors for Multivariate Kernel Density Estimation, Bulletin of the Australian Mathematical Society, 71(2), 351-352. https://doi.org/10.1017/s0004972700038314.

9. Dabral P. P., Saring, T., and Jhajharia D. (May 2016). Time Series Models of Monthly Rainfall and Temperature to Detect Climate Change for Jorhat (Assam), India. Global Nest Journal, Vol 18, No X, Pp Xx-Xx.

10. Gleim, A. and Salish, N. (2015). Forecasting Methods for Functional Time Series.

11. González, J. P. (April 2017). Functional Time Series Forecasting in Electricity Markets: ANovel Parametric

Approach.https://repositorio.comillas.edu/jspui/bitstream/11531/20872/1/TD00279.pdf.

12. Hyndman, R. J., and Shang, H. L. (2019). Functional Time Series Analysis, Package 'ftsa'. Version 5.5.

13. Hyndman, R. J., O'Hara-Wild, M., Bergmeir, C., Razbash, S. and Wang, E. (September 2017). Forecasting Functions for Time Series and Linear Models, Package 'forecast'. Version 8.2.

14. Hyndman, R. J. (2014). Forecast: Forecasting Functions of Time Series Models. R. Package, Version 7.1.

15. Hyndman, R. J., and Shang, H. L. (2010). Rainbow Plots, Bagplots, and Boxplots for Functional Data. Journal of Computational and Graphical Statistics, 19(1), 2945.https://doi.org/10.1198/jcgs.2009.08158.

16. Hyndman, R. J. and Khandakar, Y. (July 2008). Automatic Time Series Forecasting: the forecast Package for $R$ ”. Journal of Statistical Software, Volume 27, Issue 3.

17. Hyndman, R. J. and Ullah, M. S. (2007). Robust Forecasting of Mortality and Fertility Rates: A Functional Data Approach. Computational Statistics \& Data Analysis, 51, 49424956.

18. Hyndman, R. J., and Koehler, A. B. (2006). Another Look at Measures of Forecast Accuracy. International Journal of Forecasting, 22, 679-688.

19. Hyndman, R. J. (1996). Computing and Graphing Highest Density Regions. The American Statistician, 50(2), 120-126.

20. Houghton, J. T. Ding, Y. Griggs, D. J. Noguer, M. van der Linden, P. J. Dai, X. Maskell, K. and Johnson, C. A. eds., (2001). Climate. Change 2001, The Scientific Basis. Cambridge University Press, 892 pp.

21. Jain, Er. G., Mallick, Dr. B. (April 2017). A Study of Time Series Models ARIMA and ETS. I.J. Modern Education and Computer Science, 57-63.

22. Jhajharia D., Roy S. and Ete G. (2007). Climate and its Variation-A Case Study of Agartala. Journal of Soil and Water Conservation,6(1), 29-37.

23. Mudelsee, M. (2010). Climate Time Series Analysis. Classical Statistical and Bootstrap Methods. ISSN: 1383-8601, ISBN: 978-90-481-9481-0. Springer N.Y.

24. Rouf, M. A., Uddin, M. K., Debsarma, S. K. and Rahman, M. M. (November 2011). Climate of Bangladesh: An Analysis of Northwestern and Southwestern Part using High Resolution Atmosphere-Ocean General Circulation Model (AOGCM). A Scientific Journal of Krishi Foundation, The Agriculturists 9(1 and 2): 143-154,ISSN-1729-5211.

25. Ramsay, J. O., Silverman, B. W. (2005). Functional Data Analysis. Second ed. Springer, New York.

26. Rousseeuw, P. J., Ruts, I. and Tukey, J. W. (1999). The Bagplot: A Bivariate Boxplot. The American Statistician, 53(4), 382-387.

27. Sarker, M. A. R., Alam, K., and Gow, J. (2019). Performance of Rain-Fed Aman Rice 
Yield in Bangladesh in the Presence of climate Change. Renewable Agriculture and Food Systems, 34(4), 304-312. https://doi.org/10.1017/S1742170517000473.

28. Shahid, S. (2010). Recent Trends in the Climate of Bangladesh. Climate Research , 42(3): 185-193.

29. Shahid, S. (2010). Rainfall Variability and the Trends of Wet and Dry Periods in Bangladesh. International Journal of Climatology, 30(15), 2299-2313. https://doi.org/10.1002/joc.2053.

30. Shahid, S., and Khairulmaini, O. S. (2009). Spatio-Temporal Variability of Rainfall over Bangladesh During the Time Period 1969-2003. Asia-Pacific Journal of Atmospheric Sciences, 43(5), 375-389.

31. Shang, H. L. and Hyndman, R. J. (2016). Rainbow Plots, Bagplots, and Boxplots for Functional Data, Package 'rainbow'. Version 3.4.

32. Shang, H. L. (September 2012). Functional Time Series Approach for Forecasting Very Short-Term Electricity Demand. Journal of Applied Statistics, Vol. 00, No. 00, 1-17, ISSN: 0266-4763, Taylor and Francis.

33. Villarini, G., Smith, J. A., Napolitano, F. (2010). Non-Stationary Modeling of a Long Record of Rainfall and Temperature over Rome. Advances in Water Resources, Volume 33(10), 12561267.

34. Yasmeen, F. (2019). Measuring Global Warming: Global and Hemisphere Mean Temperature Anomalies Predictions using Sliced Functional Time Series (SFTS) Model. Open Journal of Applied Sciences, 09(05), 316-334. https://doi.org/10.4236/ojapps.2019.95026.

35. Yasmeen, F., and Hameed, S. (2018). Forecasting of Rainfall in Pakistan via SlicedFunctional Time Series (SFTS). World Environment, 8(1), 1-14. https://doi.org/10.5923/j.env.20180801.01.

36. Yasmeen, F. (August 2016). Modeling Breast Cancer Incidence Rates: A Comparison between the Components of Functional Time Series (FTS) Model Applied on Karachi (Pakistan) and US Data. Open Journal of Applied Sciences, 524-533, Scientific Research Publishing.

37. Yamoah, A., E., Bashiru I. Saeed and Karim, A. (2016). Sarima Modelling and Forecasting of Monthly Rainfall in the Brong Ahafo Region of Ghana. World Environment, 6(1), 1-9.

38. Yasmeen, F., and Sharif, M. (2015). Functional Time series (FTS) Forecasting of Electricity Consumption in Pakistan. International Journal of Computer Applications, 124(7), 15 19. https://doi.org/10.5120/ijca2015905518.

39. Yasmeen, F. and Zaheer, S. (2014). Functional Time Series Models to Estimate Future Age Specific Breast Cancer Incidence Rates for Women in Karachi, Pakistan. Journal of Health Science, 2, 213-221.

40. Yasmeen, F. and Mughal, S. (2014). Functional Time Series Models and the APC Models: A Comparative Study on the Lung Cancer Incidence Rates in Denmark. Journal of US-China Medical Science, David Publishing Company, 11 (3) pages. 121-128

41. Yasmeen, F. Fatima, H. and Mahmood, Z. (2014). An FDA Approach to Forecast AgeSpecific Fertility Rates of Pakistan Region-Wise. Computer Science and Applications, Ethan Publishing Company 1(6) pages. 341-348. 